\title{
DETERMINAÇÃO DE Fe E Zn EM QUEIJOS PETIT SUISSE: ASPECTOS ANALÍTICOS E TEORES ENCONTRADOS EM AMOSTRAS COMERCIAIS
}

\section{Determination of $\mathrm{Fe}$ and $\mathrm{Zn}$ in Petit Suisse cheese: analytical aspects and levels found in commercial samples}

\author{
Rafael Arromba Sousa ${ }^{2 *}$, Thalles Pedrosa Lisboa ${ }^{l}$
}

\begin{abstract}
RESUMO
O Petit Suisse é um queijo bastante popular entre o público infantil, apresentase geralmente enriquecido com as vitaminas D e E, além de sais minerais de cálcio, fósforo, zinco e ferro. Neste trabalho avaliaram-se criticamente os teores de Fe e $\mathrm{Zn}$ nesses queijos empregando a espectrometria de absorção atômica em chama (F AAS) e diferentes métodos de mineralização das amostras (via seca e via úmida). O método por via úmida, que foi adaptado para as amostras estudadas, forneceu resultados mais exatos e foi utilizado para análise de nove amostras comerciais, cujos teores de Fe variaram em uma faixa de concentração de $0,010-0,066 \mathrm{mg} \mathrm{g}^{-1}$ enquanto os teores de $\mathrm{Zn}$ ficaram entre $0,004-0,057 \mathrm{mg} \mathrm{g}^{-1}$. A presença destes micronutrientes também foi constatada em amostras comerciais não enriquecidas, portanto a qualidade do leite é de essencial importância nas características do produto final. O método proposto por via úmida é sensível o suficiente para determinação destes micronutrientes minerais.
\end{abstract}

Palavras-chave: Petit Suisse; queijo; minerais; absorção atômica.

\begin{abstract}
The Petit Suisse cheese is very popular among children, it is usually enriched with vitamins D and E and also enriched with calcium, phosphorus, zinc and iron salts. In this work it was critically evaluated the levels of Fe and $\mathrm{Zn}$ in commercial petit suisse cheeses employing the flame atomic absorption spectrometry (FAAS) and

1 Universidade Federal de Juiz de Fora, Departamento de Química, Rua José Lourenço Kelmer, s/n, Campus Universitário, São Pedro, 36036-900, Juiz de Fora, MG, Brasil. E-mail: rafael.arromba@gmail.com

* Autor para correspondência.
\end{abstract}


different methods of mineralization of the samples (dry ashing and wet digestion). The wet digestion, which was selected for the studied samples, provided results that are more accurate and then was used to analyze nine commercial samples which levels of Fe varied in a concentration range from 0.010 to $0.066 \mathrm{mg} \mathrm{g}^{-1}$ while the levels of $\mathrm{Zn}$ ranged from 0.004 to $0.057 \mathrm{mg} \mathrm{g}^{-1}$. The presence of these micronutrients was also observed in non-enriched commercial samples which shows that the quality of milk is of key importance in the final product characteristics. The method is sensitive enough to determine these mineral micronutrients.

Keywords: Petit Suisse; cheese; minerals; atomic absorption.

\section{INTRODUÇ̃̃O}

O Petit Suisse é um queijo fresco não maturado que pode ser adicionado de outras substâncias alimentícias como polpas, corantes e aromas (BRASIL, 2001). Desenvolvido por volta de 1850 na região da Normandia por Charles Chervais, tornouse bastante popular entre o público infantil desde seu lançamento no mercado brasileiro, na década de 70 (DANONE, 2014). Nos anos seguintes a formulação do produto foi alterada e este começou a ser comercializado com a adição de cálcio, ferro e zinco.

As exigências de um mercado alimentício diferenciado vêm abrindo "portas" para o consumo de alimentos enriquecidos/fortificados, como são algumas marcas de Petit Suisse e, hoje em dia, encontram-se disponíveis diversos produtos que apresentam em sua composição a adição de nutrientes como vitaminas e/ou minerais. Logo, a introdução destes componentes em alimentos mostra a potencialidade de obtenção de produtos com aspectos nutricionais mais elevados, que são destinados a públicos específicos e cada vez mais conscientes (PRUDENCIO, 2006; CARDARELLI et al., 2007).

No Brasil considera-se fortificado todo alimento ao qual é adicionado um ou mais nutrientes essenciais, contidos no alimento naturalmente ou não, com o objetivo de reforçar seu valor nutricional e/ou prevenir, ou mesmo corrigir deficiências nutricionais.
Tecnicamente, o termo enriquecimento ou fortificação é permitido quando $100 \mathrm{~g}$ do produto, pronto para consumo, fornecem no mínimo $30 \%$ da ingestão diária recomendada (IDR) de referência no caso de alimentos sólidos (ANVISA, 1998).

Em sua forma comercial o queijo Petit Suisse apresenta-se geralmente enriquecido com as vitaminas D e E, além de sais dos minerais já citados.

Embora os produtos fortificados mostrem-se como excelentes alternativas para o combate à subnutrição, a monitoração desses níveis de fortificação também é importante do ponto de vista nutricional, visto que alguns micronutrientes minerais tornam-se tóxicos, quando ingeridos em excesso (FDA, 2001; GARCÍA-RICO et al, 2007) e, que, muitos dos alimentos destinados ao público infantil são fortificados com minerais e vitaminas.

Além disso, uma vez que produtos lácteos são consumidos por todas as faixas etárias, por serem considerados boas fontes de vitaminas e minerais, a real necessidade de fortificação precisa ser estudada.

Considerando que ainda não se tem um método analítico que seja comprovadamente adequado para queijos Petit Suisse, almejouse estudar um método capaz de realizar as determinações de $\mathrm{Fe}$ e $\mathrm{Zn}$ em queijos Petit Suisse com emprego de diferentes métodos de mineralização das amostras (via seca e via úmida), prezando a praticidade, rapidez e eficiência. 


\section{MATERIAL E MÉTODOS}

Inicialmente, procedimentos de mineralização de amostras foram realizados por via seca segundo o método descrito pelo Instituto Adolfo Lutz (AOAC, 1995; JOINT FAO, 1999; IAL, 2008) e, posteriormente, empregando um método por via úmida que foi adaptado de KHOUZAM e colaboradores (KHOUZAM et al., 2011). Esses métodos foram testados e estatisticamente comparados em termos de exatidão e precisão. Considerando-se os teores esperados para os analitos, a técnica analítica utilizada foi a espectrometria de absorção atômica em chama (F AAS), que possibilitou detectar e quantificar os analitos em 9 amostras comerciais.

\section{Amostras}

As amostras de queijo Petit Suisse foram adquiridas no comércio de Juiz de Fora, sendo obtidas 9 amostras, dentre três marcas. Duas dessas marcas são comercializadas sob a forma enriquecida com os analitos de interesse e estas marcas foram designadas como "E-1" e "E-2". Já a terceira marca obtida é comercializada sob a forma tradicional, ou seja, sem fortificação e foi designada como amostra "T".

\section{Equipamentos e reagentes}

Foram utilizados os seguintes equipamentos: espectrômetro de absorção atômica com chama (VARIAN, modelo Fast Sequential Atomic Absorption Spectrometer-AA280FS), chapa elétrica de aquecimento (IKA ${ }^{\circledR} \mathrm{C}-\mathrm{MAG}$ HP10) e forno mufla (JUNG).

Para o preparo das amostras e soluções padrão para calibração do equipamento de absorção atômica utilizou-se água deionizada e reagentes de grau analítico, dentre estes ácido nítrico $\left(\mathrm{HNO}_{3}\right.$ P.A. Vetec, $\left.65 \% \mathrm{v} / \mathrm{v}\right)$ e peróxido de hidrogênio $30 \% \mathrm{~m} / \mathrm{m}$ (Vetec).
Soluções padrão multielementares de $\mathrm{Fe}$ e $\mathrm{Zn}$ foram preparadas em $\mathrm{HNO}_{3} 2 \% \mathrm{v} / \mathrm{v}$ a partir de padrões monoelementares de $1000 \mathrm{mg} \mathrm{L}^{-1}$ de Fe (Merck - Titrisol ${ }^{\circledR}$ ) e Zn (Qhemis High Purity).

Além disso, todos os materiais de uso laboratorial empregados foram de vidro ou plástico e, antes do uso, foram descontaminados em banho de ácido nítrico a $10 \% \mathrm{v} / \mathrm{v}$ por pelo menos 12 horas e, após, enxaguados com água deionizada.

\section{Preparo de amostras}

O procedimento de mineralização ocorreu por dois métodos distintos: via seca e via úmida. Em ambos os métodos as amostras foram utilizadas tal qual são comercializadas, ou seja, no estado in natura. Para avaliação da exatidão dos métodos realizou-se ensaios de adição e recuperação de analito, adicionando alíquotas conhecidas de um padrão multielementar de Fe e Zn às amostras, logo após a pesagem das mesmas. Assim, por meio da análise de amostras adicionadas dos analitos e comparando o resultado com o de amostras não adicionadas, bem como se considerando o teor dos analitos esperados (em função da adição), pôdese estimar o erro da análise, expresso em porcentagem de recuperação. Logo, quanto maior a porcentagem de recuperação obtida, menor o erro, mais exato se mostra o método e, consequentemente, mais confiável é o resultado analítico.

\section{Mineralização por via seca}

Pesou-se aproximadamente $500 \mathrm{mg}$ de amostra úmida em cadinhos de porcelana, sequencialmente submetidos a préaquecimento a $105{ }^{\circ} \mathrm{C}$ por 2 horas em forno mufla. Após este período elevou-se a temperatura a $525{ }^{\circ} \mathrm{C}$ por 14 horas. Ao final deste processo observaram-se cinzas 
brancas no fundo dos cadinhos e estas foram dissolvidas em solução de $\mathrm{HNO}_{3} 2 \%$ v/v, transferindo-se os digeridos para balões volumétricos de $25 \mathrm{~mL}$.

\section{Mineralização por via úmida}

Pesou-se aproximadamente $500 \mathrm{mg}$ de amostra úmida, diretamente sobre um béquer de $50 \mathrm{~mL}$. Esta foi digerida em chapa de aquecimento, sob refluxo (com auxílio de um vidro de relógio) e utilizando dois procedimentos bastante usuais: o primeiro com adições de $\mathrm{HNO}_{3} 65 \%$ v/v; e, o segundo com adições de mistura (4:1) de $\mathrm{HNO}_{3} 65 \% \mathrm{v} / \mathrm{v}$ e $\mathrm{H}_{2} \mathrm{O}_{2} 30 \% \mathrm{~m} / \mathrm{m}$, sendo que em ambos os procedimentos a temperatura empregada variou entre $85{ }^{\circ} \mathrm{C}$ e $90{ }^{\circ} \mathrm{C}$. A adição dos reagentes foi repetida sucessivas vezes (em média, cinco vezes) até que o desprendimento de vapores nitrosos (de coloração marrom) não fosse mais observado. Ao final dos processos, os digeridos obtidos foram transferidos para balões volumétricos de $25 \mathrm{~mL}$ e os volumes foram completados com água deionizada.

\section{Determinação dos analitos por espec- trometria de absorção atômica em chama}

Os teores de Fe e $\mathrm{Zn}$ foram determinados utilizando-se um espectrômetro de absorção atômica em chama (F AAS). As análises foram realizadas em condições instrumentais baseadas nas especificações fornecida pelo fabricante do equipamento e que são adequadas para amostras aquosas, visto que os digeridos foram diluídos em ácido nítrico diluído (mineralização por via seca) e água deionizada (mineralização por via úmida).

Utilizou-se um padrão analítico de Fe (Merck) com concentração de $6,0 \mathrm{mg} / \mathrm{L}$ para ajustar as condições da chama de ar/acetileno (vazão de ar de 13,5 L/min, vazão de acetileno de 2,0 L/min e altura de $13,5 \mathrm{~mm}$ para o queimador). A determinação de ferro foi realizada com uma lâmpada de catodo oco de Fe, operando no comprimento de onda de $248 \mathrm{~nm}$. No equipamento foi usada uma abertura de fenda de $0,2 \mathrm{~nm}$ e correção de fundo com lâmpada de deutério. Para determinações de Zn, o ajuste da chama de ar/acetileno foi feito utilizando-se um padrão de Zn na concentração de 30,0 mg/L, obtendo-se uma chama nas mesmas condições empregadas para as determinações de Fe. A lâmpada de catodo oco utilizada foi de $\mathrm{Zn}$, no comprimento de onda de $213,9 \mathrm{~nm}$ e as medidas foram feitas com abertura de fenda de 1,0 nm e correção de fundo com lâmpada de deutério.

\section{RESULTADOS E DISCUSSÃO}

\section{Métodos de preparo das amostras}

\section{Mineralização por via seca}

O método utilizado foi escolhido por ser empregado pelo Laboratório de Composição de Alimentos do Departamento de Nutrição da Universidade Federal de Juiz de Fora para análise de queijos (PEREIRA, 2014). Experimentalmente, não foram observadas limitações para as amostras em questão e, visualmente, as cinzas obtidas indicaram que o procedimento foi eficiente. Na Tabela 1 são apresentados os resultados analíticos para duas amostras, uma enriquecida e aquela não enriquecida (tradicional).

Os resultados da Tabela 1 mostram as concentrações encontradas para $\mathrm{Fe}$ e $\mathrm{Zn}$ nas duas amostras, sendo que o $\mathrm{Zn}$ não foi encontrado na amostra T. Em linhas gerais, estes resultados estão coerentes com o esperado, visto que a amostra E-1 é enriquecida com os elementos em questão; entretanto, considerando os resultados obtidos para os testes de adição e recuperação de analito (amostras E1 fortificada e $\mathrm{T}$ fortificada), 
notou-se que apenas para $\mathrm{Fe}$, na amostra E-1, foi obtida uma boa recuperação. Isto indica que o método não foi robusto o suficiente para ambos os analitos nessas duas amostras (enriquecida e tradicional).

Neste sentido, deve-se considerar que o aquecimento excessivo em mineralizações por via seca pode levar à perda de analito por volatilização (IAL, 2008) ou, ainda, que este tipo de queijo, por apresentar uma alta umidade pode ter parte do seu material projetado (ocasionando a perda de analito) quando submetido ao procedimento a altas temperaturas. Em contrapartida, vale ressaltar que o uso de temperaturas relativamente baixas pode não ser capaz de proporcionar uma mineralização completa da amostra. Sendo assim, como o procedimento descrito na literatura para queijos em geral não se mostrou adequado neste caso, ficando evidente a necessidade de otimizá-lo para o Petit Suisse, optou-se por estudar um método de mineralização por via úmida fazendo-se uso de condições menos drásticas e mais controladas.

\section{Mineralização por via úmida (digestão ácida)}

Os digeridos obtidos a partir dos métodos de mineralização empregados (digestão com ácido nítrico e digestão com ácido nítrico e peróxido de hidrogênio) foram solubilizados em água deionizada e analisados por F AAS nas condições selecionadas e especificadas no Material e Métodos. Os resultados obtidos estão apresentados na Tabela 2 e foram comparados estatisticamente por meio dos testes $\mathrm{F}$ e $t$, ambos no nível de $95 \%$ de confiança.

A partir dos resultados obtidos para determinação dos analitos foi feito o teste $t$-pareado. Constatou-se que para a determinação de Fe o $t$ calculado $(2,224)$ apresentou-se menor que o $t$ tabelado (2.776) e que para a determinação de $\mathrm{Zn}$ o mesmo comportamento foi obtido: o t calculado $(2,159)$ foi menor que o $t$ tabelado $(2,776)$.

Como se observou que os valores de t calculado foram menores que os valores de

Tabela 1 - Concentrações de Fe e Zn para amostras enriquecida e tradicional, tal qual comercializadas e para as mesmas amostras, depois de fortificadas com os analitos, para avaliação da exatidão do método

\begin{tabular}{|c|c|c|}
\hline \multicolumn{3}{|c|}{ Determinação de Fe } \\
\hline Amostras & Concentração em $\mathrm{mg} \mathrm{g}^{-1}$ & \% de Recuperação \\
\hline E-1 & $0,037 \pm 0,005$ & \multirow{2}{*}{34} \\
\hline E-1 Fortificada & $0,044 \pm 0,008$ & \\
\hline $\mathrm{T}$ & $0,053 \pm 0,023$ & \multirow{2}{*}{104} \\
\hline T Fortificada & $0,096 \pm 0,005$ & \\
\hline \multicolumn{3}{|c|}{ Determinação de $\mathbf{Z n}$} \\
\hline Amostras & Concentração em $\mathrm{mg} \mathrm{g}^{-1}$ & \% de Recuperação \\
\hline E-1 & $0,017 \pm 0,003$ & \multirow{2}{*}{7,7} \\
\hline E-1 Fortificada & $0,019 \pm 0,003$ & \\
\hline $\mathrm{T}$ & ND* & \multirow{2}{*}{55} \\
\hline T Fortificada & $0,011 \pm 0,006$ & \\
\hline
\end{tabular}

(*) ND = não detectado 
t tabelado conclui-se que para a determinação de Fe e Zn não há evidências de diferenças significativas entre os dois métodos de digestão das amostras de queijo Petit Suisse. Dessa forma, optou-se por prosseguir o estudo analítico com a utilização do método empregando apenas $\mathrm{HNO}_{3}$, que se mostrou suficiente para oxidar a matéria orgânica no processo de digestão.

\section{Validação analítica e aplicação do método}

Utilizando o método de digestão definido (apenas $\mathrm{HNO}_{3}$ e aquecimento em chapa), amostras de três marcas diferentes de queijo Petit Suisse foram avaliadas: E-1 e E-2, que são do tipo enriquecidas e a amostra $T$, não enriquecida. Nessas amostras foram determinados os teores de Fe e Zn, para os quais foi possível estabelecer uma faixa de concentração. Um resumo dos resultados obtidos, bem como alguns dos parâmetros de mérito do método (limites de detecção, quantificação e exatidão) estão apresentados na Tabela 3 .
Considerando a obtenção dos dados analíticos resumidos na Tabela 3, pôde-se constatar que o método de análise aplicado comportou-se como uma ferramenta prática, rápida e eficaz para determinação de $\mathrm{Fe}$ e $\mathrm{Zn}$ em amostras comerciais de queijo Petit Suisse, apresentando detectabilidade adequada e boa exatidão. Esta última foi avaliada por meio de testes de adição e recuperação do analito, do mesmo modo que o utilizado no estudo do método da mineralização por via seca; entretanto, apresentou um melhor resultado quando as amostras foram preparadas por meio dessa digestão ácida.

Quanto à aplicação do método para estudar amostras comerciais, as faixas de concentração de Fe e Zn observadas na Tabela 3 , indicam que a fortificação em E-2 acontece em maior percentual que em E-1, porém esta diferença está coerente com as informações declaradas nos rótulos de ambas as marcas, embora diferenças de até $128 \%$ foram encontradas entre os valores declarados e os encontrados experimentalmente. Nota-se também que a amostra

Tabela 2 - Valores médios em $\mathrm{mg} \mathrm{g}^{-1}$ de Fe e $\mathrm{Zn}$ para os diferentes métodos de digestão

\begin{tabular}{ccc}
\hline \multicolumn{3}{c}{ Determinação de Fe } \\
\hline Ensaio & Adição apenas de $\mathrm{HNO}_{3}$ & Adição de $\mathrm{HNO}_{3}+\mathrm{H}_{2} \mathrm{O}_{2}$ \\
\hline 1 & 0,027 & 0,031 \\
2 & 0,029 & 0,030 \\
3 & 0,028 & 0,028 \\
4 & 0,042 & 0,050 \\
5 & 0,046 & 0,054 \\
\hline \multicolumn{3}{c}{} \\
\hline Ensaio & Adição apenas de $\mathrm{HNO}_{3}$ & Adição de $\mathrm{HNO}_{3}+\mathrm{H}_{2} \mathrm{O}_{2}$ \\
\hline 1 & 0,015 & 0,017 \\
2 & 0,016 & 0,029 \\
3 & 0,018 & 0,018 \\
4 & 0,027 & 0,031 \\
5 & 0,030 & 0,035 \\
\hline
\end{tabular}


$\mathrm{T}$ (tradicional), sem enriquecimento com esses nutrientes, apresenta naturalmente em sua composição os micronutrientes Fe e Zn, provavelmente oriundos do leite utilizado em sua fabricação, que é conhecido por ser fonte dos oligoelementos ferro e zinco entre outros (VERRUMA; SALGADO, 1994; KIRA, 2002), onde se destaca o papel do zinco associado a partículas de caseinato (GONZÁLEZ, 2001).

Em relação aos teores encontrados para os micronutrientes, dentre as diferentes amostras, as faixas de concentração correspondentes são amplas e sugerem que não existe um padrão de concentração bem definido para os teores de Fe e Zn. Isto pode estar relacionado com o fato desses micronutrientes já estarem presentes na matéria-prima (evidenciado pelos resultados da amostra "T"), cuja variação influencia a variação final do produto fortificado.

Considerando este aspecto e o fato de que muitos outros produtos destinados ao público infantil também são fortificados com micronutrientes, talvez não seja necessária utilizar a fortificação em produtos que já contém os nutrientes, tendo em vista que tais micronutrientes podem vir a ser ingeridos em excesso, levando-se em conta a contribuição de toda uma dieta.

\section{CONCLUSÕES}

O método tradicional para abertura de amostras (mineralização por via seca), recomendado pela literatura, mostra-se ineficaz e

Tabela 3 - Resultados obtidos para determinação de Fe e Zn em amostras de queijo Petit Suisse

\begin{tabular}{ccc}
\hline \multicolumn{3}{c}{ Determinações de Fe } \\
\hline Amostras & Faixa de concentração em $\mathrm{mg} \mathrm{g}^{-1}$ & Desvio padrão relativo \\
\hline E-1 & $0,032-0,033$ & $1,10-2,67$ \\
$\mathrm{E}-2$ & $0,062-0,066$ & $0,27-4,73$ \\
$\mathrm{~T}$ & $0,010-0,012$ & $1,79-2,32$ \\
\hline \multirow{2}{*}{ Limites } & Limite de detecção $(L D)$ em $m g g^{-1}$ & Limite de quantificação (LQ) em $\mathrm{mg} \mathrm{g}^{-1}$ \\
& 0,0001 & 0,0004 \\
\cline { 2 - 3 } Exatidão & $E-1$ & $T$ \\
& Erro $\leq 3 \%$ & Erro $\leq 9 \%$ \\
\hline
\end{tabular}

\section{Determinações de $\mathbf{Z n}$}

\begin{tabular}{ccc}
\hline Amostras & Faixa de concentração em $\mathrm{mg} \mathrm{g}^{-1}$ & Desvio padrão relativo \\
\hline E-1 & $0,014-0,034$ & $1,06-9,54$ \\
E-2 & $0,054-0,057$ & $0,66-1,80$ \\
T & $0,004-0,006$ & $4,06-6,94$ \\
\hline
\end{tabular}

Limites

Limite de detecção (LD) em $\mathrm{mg} \mathrm{g}^{-1} \quad$ Limite de quantificação (LQ) em $\mathrm{mg} \mathrm{g}^{-1}$

\begin{tabular}{ccc} 
& 0,0016 & 0,0053 \\
\cline { 2 - 3 } Exatidão & $E-1$ & $T$ \\
Erro $\leq 12 \%$ & Erro $\leq 11 \%$
\end{tabular}

$\mathrm{LD}=3 \times \mathrm{xPa}$

$\mathrm{LQ}=10 \mathrm{xDPa}$

$\mathrm{DPa}=$ desvio padrão do branco amostral, $\mathrm{n}=10$ 
susceptível a contaminação (o tratamento todo leva mais de 12 horas).

A aplicação da digestão ácida ao conjunto das nove amostras estudadas, dentre produtos fortificados e não fortificados (tradicional), fornece resultados satisfatórios em termos de exatidão e detectabilidade, permitindo caracterizar as amostras analisadas em relação aos teores desses micronutrientes.

Como perspectivas futuras para a continuidade deste trabalho considera-se importante estudar outros produtos enriquecidos com Fe e $\mathrm{Zn}$ para que se possa estimar a ingestão diária de indivíduos que consomem tais produtos com regularidade e, assim, avaliar a real necessidade desses enriquecimentos.

\section{AGRADECIMENTOS}

À FAPEMIG e à Rede Mineira de Química, pelo apoio financeiro e à Votorantim Metais Juiz de Fora, na pessoa do Marcelo Biolcati, pelo auxílio na realização das análises químicas.

\section{REFERÊNCIAS}

AGÊNCIA NACIONAL DE VIGILÂNCIA SANITÁRIA (ANVISA). Portaria $n^{\circ} 31$ de 13 de janeiro de 1998. Aprova o Regulamento Técnico para Fixação de Identidade e Qualidade de Alimentos Adicionados de Nutrientes Essenciais. Diário Oficial da República Federativa do Brasil, Brasília, 16 jan. 1998.

ASSOCIATION OF ANALYTICAL CHEMISTS (AOAC). Official Methods of Analysis. 16th ed. Washington: AOAC, 1995.

BRASIL. Ministério da Agricultura, Pecuária e Abastecimento. Instrução Normativa $\mathrm{n}^{\circ}$ 53, de 29 de dezembro de 2000. Aprova o Regulamento Técnico de Identidade e Qualidade de Queijo “Petit Suisse”. Diário
Oficial da República Federativa do Brasil, Brasília, 04 jan. 2001. Seção 1, p. 3

CARDARELLI, H. R. et al. Functional petitsuisse cheese: Measure of the prebiotic effect. Anaerobe, v. 13, n. 5-6, p. 200-207, 2007.

DANONE. Tudo Sobre Iogurte - História do Iogurte. Disponível em: <http://www. danone.com.br/tudo-sobre-iogurte/historiado-iogurte-no-brasil/> Acesso em: 23 jul. 2014.

FOOD AND DRUG ADMINISTRATION (FDA). Dietary Reference Intakes for Vitamin A, Vitamin K, Arsenic, Boron, Chromium, Copper, Iodine, Iron, Manganese, Nickel, Silicon, Vanadium and Zinc. Washington DC: Report of the Panel on Micronutrients. National Academy Press: Washington, 2001. 800 p.

GARCÍA-RICO, L.; LEYVA-PEREZ, J.; JARA-MARINI, M. E. Content and daily intake of copper, zinc, lead, cádmium and Mercury from dietary supplements in Mexico. Food and Chemical Toxicology, v. 45, n. 9, p. 1599-1605, 2007.

GONZÁlEZ, F. H. D. Composição Bioquímica do Leite e Hormônios da Lactação. IN: GONZÁLEZ, F. H. D; DÜRR, J. W.; FONTANELI, R. S. Uso do leite para monitorar a nutrição e o metabolismo de vacas leiteiras. Porto Alegre: UFRGS, 2001. cap. 1, p. 5-22.

INSTituto ADOLFO LUTZ. Métodos físico-químicos para análise de alimentos. $4^{\mathrm{a}}$ ed., $1^{\mathrm{a}}$ ed. digital. São Paulo: Instituto Adolfo Lutz, 2008. 1020 p.

JOINT FAO, WHO FOOD STANDARDS PROGRAMME. Codex Alimentarius Commission. CODEX STAN 234: Recommended 
Methods for Analysis and Samplying, Roma, 1999.

KHOUZAM, R. B.; POHL, P.; LOBINSKI, R. Bioacccessibility of essential elements from white cheese, bread, fruit and vegetables. Talanta, v. 86, p. 425-428, 2011.

KIRA, C. S. Estudo da composição mineral e dos elementos-traço essenciais em amostras de leite e produtos lácteos por espectrometria de emissão atômica com plasma induzido e análise por ativação com nêutrons. 2002. 96 f. Dissertação (Mestrado em Ciências na Área de Tecnologia Nuclear-Aplicações) - Autarquia Associada à Universidade de São Paulo, São Paulo, 2002.

PEREIRA, J. P. F. Partição do cálcio em queijo Minas Padrão e sua bioacessibilidade ao longo do tempo de maturação. 2014. $71 \mathrm{f}$. Dissertação (Mestrado Profissional em Ciência e Tecnologia do Leite e Derivados) Universidade Federal de Juiz de Fora, Juiz de Fora, 2014.

\section{PRUDENCIO, I. D. Propriedades físicos} de queijo petit suisse elaborado com retentado de soro de queijo e estabilidade de Antocianinas e Betalaínas adicionadas. 2006. 87 f. Dissertação (Mestrado em Ciência dos Alimentos) - Universidade Federal de Santa Catarina, Florianópolis, 2006.

VERRUMA, M. R.; SALGADO, J. M. Análise Química do Leite de Búfala em Comparação ao Leite de Vaca. Scientia Agricola, v. 51, n. 1, p. 131-137, 1994. 